\title{
The Characteristics of Adults with Severe Hearing Loss
}

DOI: $10.3766 /$ jaaa. 17050

\author{
Pamela Souza* \\ Eric Hoover $\dagger$ \\ Michael Blackburn $\ddagger$ \\ Frederick Gallun§
}

\begin{abstract}
Background: Severe hearing loss impairs communication in a wide range of listening environments. However, we lack data as to the specific objective and subjective abilities of listeners with severe hearing loss. Insight into those abilities may inform treatment choices.

Purpose: The primary goal was to describe the audiometric profiles, spectral resolution ability, and objective and subjective speech perception of a sample of adult listeners with severe hearing loss, and to consider the relationships among those measures. We also considered the typical fitting received by individuals with severe loss, in terms of hearing aid style, electroacoustic characteristics, and features, as well as supplementary device use.
\end{abstract}

Research Design: A within-subjects design was used.

Study Sample: Participants included 36 adults aged 54-93 yr with unilateral or bilateral severe hearing loss.

Data Collection and Analysis: Testing included a full hearing and hearing aid history; audiometric evaluation; loudness growth and dynamic range; spectral resolution; assessment of cochlear dead regions; objective and subjective assessment of speech recognition; and electroacoustic evaluation of current hearing aids. Regression models were used to analyze relationships between hearing loss, spectral resolution, and speech recognition.

Results: For speech in quiet, $60 \%$ of the variance was approximately equally accounted for by amount of hearing loss, spectral resolution, and number of dead regions. For speech in noise, only a modest proportion of performance variance was explained by amount of hearing loss. In general, participants were wearing amplification of appropriate style and technology for their hearing loss, but the extent of assistive technology use was low. Subjective communication ratings depended on the listening situation, but in general, were similar to previously published data for adults with mild-to-moderate loss who did not wear hearing aids.

Conclusions: The present data suggest that the range of abilities of an individual can be more fully captured with comprehensive testing. Such testing also offers an opportunity for informed counseling regarding realistic expectations for hearing aid use and the availability of hearing assistive technology.

Key Words: auditory rehabilitation, hearing science, speech perception

Abbreviations: PTA = pure-tone average; SD = standard deviation; SNR = signal-to-noise ratio; $S S Q=$ Speech and Spatial Qualities; STM = spectrotemporal modulation; TEN = Threshold Equalizing Noise; WDRC $=$ wide-dynamic range compression

*Department of Communication Sciences and Disorders and Knowles Hearing Center, Northwestern University, Evanston, IL; †Auditory \& Speech Sciences Laboratory, University of South Florida, Tampa, FL; $¥$ Veterans Administration Medical Center, St. Cloud, MN; §National Center for Rehabilitative Auditory Research, Portland VA Medical Center and Oregon Health Sciences University, Portland, OR

Corresponding author: Pamela Souza, Department of Communication Sciences and Disorders, Northwestern University, Evanston, IL 60208; Email: p-souza@northwestern.edu

This work was supported by the National Institutes of Health (R01 DC006014).

A portion of these data were presented at the 2014 meeting of the American Auditory Society, Scottsdale, AZ. 
$\mathrm{S}$ evere hearing loss is simultaneously one of the most interesting and most challenging auditory problems to treat. Unlike individuals with milder degrees of loss, who have acceptable speech recognition in most environments once appropriate amplification is provided (Kochkin, 2011), listeners with severe loss may struggle to communicate in everyday situations. Without amplification, conversational-level speech will be inaudible or nearly inaudible. Even with well-fit hearing aids which improve audibility, individuals with severe loss report difficulty understanding speech in quiet and in noise (Flynn et al, 1998). The inability to obtain enough auditory input increases the burden on compensatory processes, including visual cues and working memory (Lyxell et al, 2003). As a result of their communication difficulty, these listeners report a high level of stress in social situations (Gevonden et al, 2015). In sum, a listener with severe hearing loss is likely to work harder but understand less.

Unlike listeners with milder amounts of hearing loss, whose communication abilities are roughly correlated with degree of loss (e.g., Humes, 2007; Souza et al, 2007), listeners with severe loss have more varied and less predictable abilities. Some studies suggest there may be as much as an $80 \%$ range about the speech-recognition score that would be predicted on the basis of audibility (Flynn et al, 1998; Seldran et al, 2011). In addition to understanding the measured speech-recognition abilities of this population, there is a notable lack of data on perceived abilities, particularly in the complex listening situations that are likely to present a substantial challenge for listeners with severe hearing loss.

Similarly, there are few data with regard to basic auditory abilities, including spectral resolution. The extent to which spectral resolution is impaired is of interest because it is assumed to be a major consequence of the cochlear damage patterns in severe loss, and to have significant consequences for speech recognition. Moreover, to the extent that broadened auditory filters create greater reliance on temporal cues, some authors have suggested avoiding signal processing techniques-such as short compression release times-that alter temporal cues (Boothroyd et al, 1988; Davies-Venn et al, 2009; Davies-Venn and Souza, 2014). If presumed frequency resolution is to be used as a basis for treatment decisions, we need data which confirm or predict the range of ability.

With regard to auditory treatment, listeners with severe loss are generally provided with modified versions of the same technology found to be beneficial for listeners with less hearing loss, or that seem reasonable choices based on assumed abilities. For example, because a severe sensorineural loss will reduce the dynamic range, wide-dynamic range compression (WDRC) amplification is implemented with more extreme compression ratios than for listeners with mild-to-moderate hearing loss (Souza and Bishop, 1999; Byrne et al, 2001; Moodie et al, 2007). And, given the expectation for poor speechin-noise understanding with severe loss, there is an assumption that any feature (such as directional microphones) that improves the signal-to-noise ratio will be helpful (Kühnel et al, 2001). However, all of the amplification strategies targeted toward listeners with severe loss are predicated on the idea of a homogenous population. That is, all listeners with severe loss are presumed to experience reduced dynamic range, have poor spectral resolution which causes them to rely on temporal information, and demonstrate poor speech-in-noise ability. Insight into the distribution of those abilities within the severe loss population could inform treatment choices.

Finally, it is not clear to what extent the available evidence is followed; that is, what amplification choices are being utilized by listeners with severe loss in everyday practice. Although more listeners with severe loss use hearing aids than any other degree-of-loss group, they are the least satisfied with the benefit provided by their hearing aids (Kochkin, 2009). The reasons for this dissatisfaction are not entirely clear. It may be that hearing aid benefit is limited by an inability to fully compensate for the damage that accompanies severe hearing loss. However, it is also possible that listeners with severe loss are being fit with products that do not align with the available evidence, and therefore are not optimally suited to the listener's abilities.

We propose that a more complete description of the abilities of this population and greater understanding of the sources of these difficulties may motivate more effective treatment. In contrast to hundreds of studies on listeners with mild-to-moderate hearing loss, a review of the literature for the past decade reveals fewer than a dozen empirical studies focused on listeners with severe hearing loss. Those individuals are present in significant numbers. Although listeners with severe loss comprise only $10 \%$ of all listeners with hearing loss (Cruickshanks et al, 1998), severe loss may be substantially higher in some populations, such as hearing aid wearers (36\%) (Kochkin, 2009) and older veterans (17\%) (CDC, 2011). The lack of evidence specific to this population means that clinical decisionmaking for adults with severe loss- those who are experiencing the greatest barriers to communication-must rely on data from adults with mild-to-moderate hearing loss. If we understood this population better, could we make more appropriate recommendations?

The current study approached this issue in two ways. The primary goal was to describe the audiometric profiles, spectral resolution ability, and objective and subjective speech perception of a sample of adult listeners with severe hearing loss, and to consider the relationships among those measures. We also considered the typical fitting received by individuals with severe loss, in terms of hearing aid style, electroacoustic characteristics, and features, as well as supplementary device use. 


\section{METHODS}

\section{Participants}

Regarding degree of loss, some authors (e.g., Van Tasell, 1993) have advocated for an evidence-based categorization which defines hearing loss according to cochlear damage patterns, rather than on an arbitrary division of a decibel range. Animal data suggest that hearing losses $<55 \mathrm{~dB}$ $\mathrm{HL}$ are due to outer hair cell loss, while those $>55 \mathrm{~dB}$ $\mathrm{HL}$ are caused by more complex cochlear pathology (Ryan and Dallos, 1975; Stebbins et al, 1979; Hamernik et al, 1989; Cheatham and Dallos, 2000). Following from this idea, participants were accepted into the present study if at least one ear met the study criteria of three-frequency pure-tone average (PTA, 0.5, 1, $2 \mathrm{kHz}$ ) of $55 \mathrm{~dB} \mathrm{HL}$ or greater. Participants were recruited by flyers distributed to local audiology practices and posted in the community and by advertisements in local newspapers. The test group included 36 adults ( 15 female), aged 54-93 yr (mean age = $79.0 \mathrm{yr}$ ). The PTA across ears ranged from 55 to $110 \mathrm{~dB}$ $\mathrm{HL}$, with a mean PTA of $71.6 \mathrm{~dB} \mathrm{HL}$ and a standard deviation (SD) of $13.2 \mathrm{~dB} \mathrm{HL}$. Audiograms are shown in Figure 1. The distribution of age and audiogram was comparable to other studies of severe loss (e.g., Flynn et al, 1998; Convery and Keidser, 2011) and was consistent with epidemiological data that a majority of listeners with severe loss are older adults (Turton and Smith, 2013). Four participants demonstrated mixed loss with significant air-bone gaps (defined as an air-bone gap $>10 \mathrm{~dB}$ at $0.5,1,2$, and/or $4 \mathrm{kHz}$ ). In all four cases, the loss could be described as predominantly sensorineural; that is, bone-conduction thresholds indicated substantial cochlear pathology. The remaining participants presented with a sensorineural loss, defined as the absence of significant air-bone gaps and immittance values within normal limits.

Some tests were administered only in one ear, as described below. In those cases, one ear was designated as the test ear according to the following criteria: (a) if only one ear fit the criterion of $\geq 55 \mathrm{~dB}$ PTA (three participants), the poorer ear was chosen; (b) if both ears fit the criterion of $\geq 55 \mathrm{~dB}$ PTA and one ear was asymmetrically so poor as to prevent sufficient audibility of the test stimuli, the better ear was chosen; (c) in cases of symmetrical hearing loss where the listener habitually wore only one hearing aid, the ear normally aided was chosen; (d) in cases of symmetrical hearing loss where the listener normally wore two hearing aids, one ear was designated as the test ear. Following these criteria, the right ear was selected as the test ear for 24 participants, and the left ear was selected for the remaining 12 participants.

All participants underwent an informed consent process, followed by extensive testing across two visits of approximately 2 hours each. That testing included a full hearing and hearing aid history; audiometric evaluation; electroacoustic evaluation of current hearing aids; loudness growth and dynamic range; spectral resolution; assessment of cochlear dead regions; objective and subjective assessment of speech recognition. Specific components of the evaluation are described below.
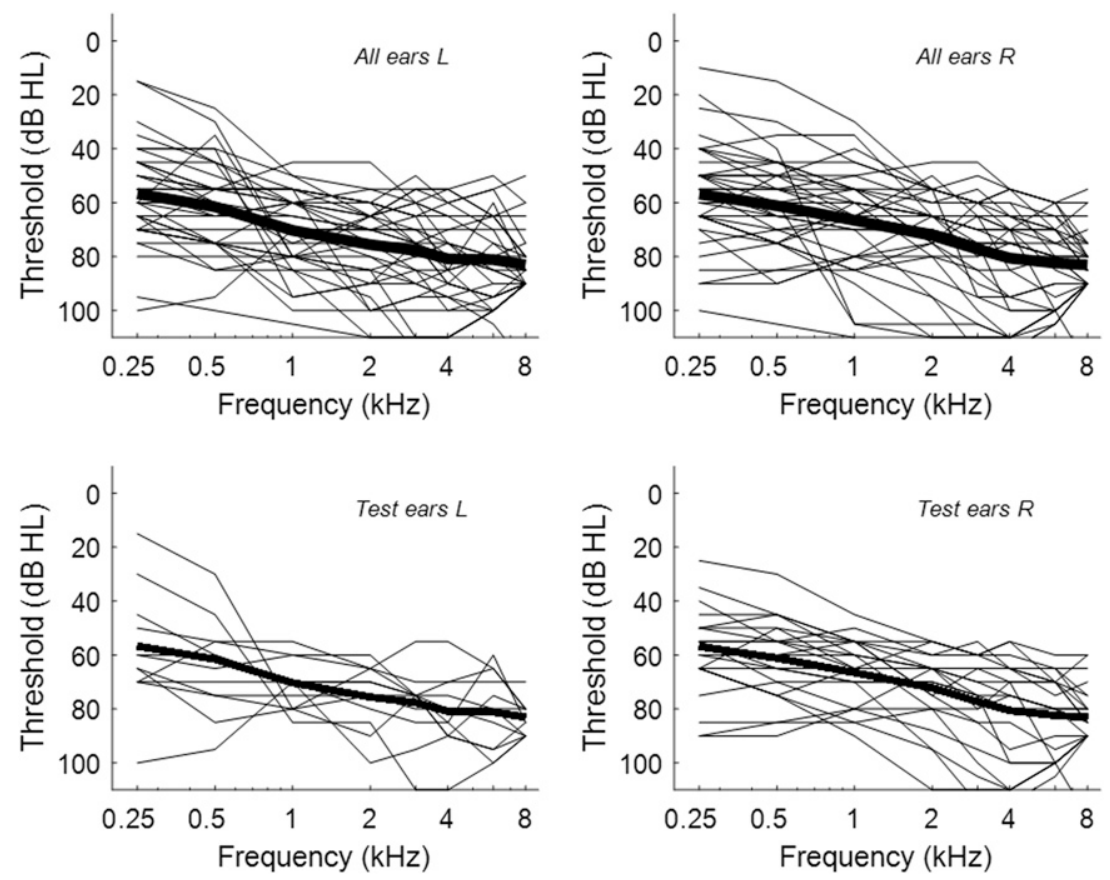

Figure 1. Pure-tone air conduction audiograms for study participants. The top panels show all left and right ear audiograms; lower panels show audiograms for the ear selected for monaural testing. Thick line in each panel shows (left or right) mean audiogram for the entire cohort. 


\section{Electroacoustic Assessment of Current Hearing Aids}

All participants' hearing aids underwent an electroacoustic analysis as follows. All measurements were completed at user settings and with the appropriate coupler (HA1 or HA2) for style of aid, using an Audioscan Verifit hearing aid test system. Coupler levels (in $\mathrm{dB}$ SPL) were measured as a function of frequency for running speech inputs of 50, 60, and $70 \mathrm{~dB}$ SPL. In each case, the input signal was the "carrot passage" (Cole, 2009) spoken by a male talker. Pure-tone input-output functions were obtained at octave frequencies from 0.25 to $4 \mathrm{kHz}$. Wide-dynamic range compression processing was considered to be present if the relative levels of coupler outputs indicated compression (i.e., $<10 \mathrm{~dB}$ difference in output with input signals of 50 and $60 \mathrm{~dB}$ SPL) or if input-output functions showed a compression threshold of $<50 \mathrm{~dB}$ SPL (Dillon, 1988).

Directivity and digital noise reduction were measured in the coupler, using the available equipment features (Audioscan, 2016). For directional hearing aids, directionality was measured by positioning the hearing aid in the sound chamber relative to a front $\left(0^{\circ}\right.$ azimuth $)$ and a rear $\left(180^{\circ}\right.$ azimuth) speaker. Speech ( $60 \mathrm{~dB}$ SPL $)$ was presented from the front speaker and noise $(60 \mathrm{~dB}$ SPL) was presented from the rear speaker. At periodic intervals, a brief noise burst was presented from either the front or rear speaker to probe the hearing aid output from that direction without influencing automatic behaviors. The output consisted of two coupler output recordings, one for each speaker. Directivity was quantified as the difference in $\mathrm{dB}$ between the magnitude spectra of the front and rear recordings.

To measure noise reduction, a $60 \mathrm{~dB}$ SPL "vacuum" signal was presented. The system measured an immediate response (quantified as $\mathrm{dB}$ SPL as function of frequency) before the hearing instrument reacted to the presence of noise. A second curve was captured after adaptive gain reduction had reached maximum effect (as determined by visual inspection of the dynamic curve). Noise reduction was quantified as the difference in $\mathrm{dB}$ between the magnitude spectra with and without adaptive gain reduction.

\section{Loudness Discomfort Levels and Dynamic Range}

Frequency-specific loudness discomfort levels were measured for both ears using a standard clinical procedure (Hawkins et al, 1987) and clinical audiometer (Grason Stadler Audiostar) coupled to insert earphones (Etymotic Research ER-3). Warble tones were presented at 0.5 and $3 \mathrm{kHz}$ in ascending presentations, using a $5 \mathrm{~dB}$ step size. The participant was asked to indicate the level at which sound became uncomfortably loud. For each ear and test frequency, loudness discomfort level was measured twice, and the final loudness discomfort level was taken as the mean across the two values.

\section{Spectral Resolution}

Spectral resolution was measured monaurally in the designated test ear. Broadband spectral resolution was quantified using the spectral ripple test (Henry et al, 2005; Won et al, 2007). The signal was a pure-tone complex comprised of a weighted sum of 800 sinusoidal components spanning the frequency range from 100 to $5000 \mathrm{~Hz}$. The amplitudes of the sinusoids were adjusted so that the resultant spectrum reproduced multiple periods of a full-wave rectified sinusoid on a logarithmic frequency scale. The peak-to-valley ratio of the ripples was fixed at $30 \mathrm{~dB}$. The signals varied according to the number of periods (or ripples) of the sinusoidal envelope present in one octave of the stimuli. Number of ripples was varied in 16 steps: $0.125,0.176,0.250,0.354,0.500,0.707,1.000$, $1.414,2.000,2.828,4.000,5.657,8.000,11.314,16$, and 22.628 ripples per octave. The ripple spectra were digitally filtered to approximate the long-term spectrum of speech, converted to analog (TDT RX6), adjusted to the desired level, and presented to the listener via an insert earphone (Etymotic Research ER-3).

To determine the appropriate presentation level for the ripple stimuli, loudness sensitivity was measured using a modified version of the Contour test (Cox et al, 1997) implemented in MATLAB (Mathworks, 2014). The stimuli were noise bursts with the same duration and bandwidth as the ripple stimuli but no spectral envelope modulation. After a single trial, the listener chose an option from the following ratings displayed on a computer screen: inaudible; very soft; soft; comfortable but slightly soft; comfortable; comfortable but slightly loud; loud but ok; and uncomfortably loud. After each trial, the stimulus level was increased by $5 \mathrm{~dB}$. Regardless of presentation level, the test terminated with a response of "uncomfortably loud." The entire sequence of ratings was repeated twice. The purpose of these loudness growth measurements was to facilitate setting of the level of the ripple stimuli.

To measure spectral resolution, the listener was asked to select the interval that was different in a three-alternative forced-choice task, also implemented in MATLAB. The stimuli were presented at a level that was "comfortable but slightly loud" as determined during the Contour test. Two intervals contained the same spectral envelope, and the target interval contained an inverted spectral envelope. To detect differences between the target and inverted spectral envelopes, it is necessary to resolve the ripple spacing. To minimize the use of perceived loudness to select the target ("different") interval, the presentation level was varied randomly from trial to trial ( $\pm 4 \mathrm{~dB}$ in $1 \mathrm{~dB}$ steps). The initial presentation was at 0.125 ripples/octave. The task varied adaptively such that the number of ripples per octave increased after two correct identifications and 
decreased after one incorrect identification. Accordingly, higher scores (more ripples/octave) represent better performance. The mean of the final eight of 12 reversals were used to calculate the threshold. At the beginning of the first block, the experimenter verified that the participant was correctly identifying the target stimulus at 0.125 ripples per octave, and that they understood the task. Each participant completed three test blocks.

\section{Cochlear Dead Regions}

Presence of cochlear dead regions was evaluated in the test ear using the Threshold Equalizing Noise (TEN) test (Moore et al, 2004). The premise of the TEN is that in areas of nonfunctioning inner hair cells, a response to pure-tones in quiet may occur because of stimulation of adjacent cochlear regions. The TEN test measures pure-tone thresholds in the presence of a frequencyshaped noise that for a listener with normal hearing will result in equivalent masked thresholds across frequency. For example, a listener presented with a $70 \mathrm{~dB}$ HL TEN noise should demonstrate thresholds of $70 \mathrm{~dB} \mathrm{HL}( \pm 5 \mathrm{~dB}$ of normal variability). If the response to the tone is actually generated at an off-frequency place, the noise will have a greater masking effect. Accordingly, a masked threshold that is $>10 \mathrm{~dB}$ above the level of the TEN noise is considered to indicate a dead region.

In this study, TEN thresholds were measured at 1, 2, and $3 \mathrm{kHz}$. The TEN level was set to $10 \mathrm{~dB}$ SL re: unmasked pure-tone threshold at the test frequency. Test procedure followed that was suggested by Moore (2010), with the threshold specified as two of three responses to ascending tone presentations, using a $2 \mathrm{~dB}$ step size. The test was considered to be positive for a dead region if the masked threshold was $>10 \mathrm{~dB}$ above the unmasked threshold at the test frequency.

\section{Objective Speech Recognition}

Measures of objective speech recognition included monosyllabic words in quiet and sentences in noise. Monosyllabic word recognition was measured using a conventional clinical approach in which a list of 50 recorded NU-6 words (Tillman and Carhart, 1966) was presented at $30 \mathrm{~dB}$ SL re: three-frequency PTA to each ear. If the listener reported loudness discomfort at $30 \mathrm{~dB}$ SL, the presentation level was reduced.

Speech-in-noise recognition was measured using the QuickSIN (Killion et al, 2004) in monaural left, monaural right and binaural conditions. Because all of our participants had PTAs exceeding $45 \mathrm{~dB} \mathrm{HL}$, presentation level was set to "loud but comfortable" as judged by the participant (per the test instructions). The listener first completed a practice list in the better ear. This was followed by two lists per condition, with list number randomly selected from equivalent lists (McArdle and Wilson, 2006) without list repetition. The final score for each condition was the mean score across two lists.

\section{Subjective Speech Recognition}

The measure of subjective speech recognition was the Speech and Spatial Qualities (SSQ) questionnaire (Gatehouse and Noble, 2004). The SSQ was selected for its emphasis on specific difficult listening situations, such as communication with multiple talkers, or when trying to follow a single talker in a background of other noise. It contains questions in three domains: speech recognition (14 questions; e.g., "You are talking with one other person in a quiet, carpeted lounge room. Can you follow what the other person says?"); spatial aspects of hearing (17 questions; e.g., "Can you tell how far away a bus or a truck is, from the sound?"); and quality aspects such as clarity and naturalness (18 questions; e.g., "When you listen to music, does it sound clear and natural?"). For each question, the participant was asked to rate their ability on a continuous scale from 0 (not at all) to 10 (perfectly). Participants responded to the questionnaire under their habitual listening condition (i.e., hearing aid wearers answered questions as for aided listening). Participants were also allowed to indicate if a question was not relevant to their experience.

\section{RESULTS}

\section{History and Etiology}

Average age of onset of loss was reported to be $49.4 \mathrm{yr}$. However, there was a wide range, from congenital loss to onset at $80 \mathrm{yr}$ of age. The majority of participants reported onset of loss after $50 \mathrm{yr}$ of age. With regard to loss progression, $78 \%$ of the participants reported their hearing loss had occurred gradually, and $22 \%$ reported a sudden loss. Probable etiologies (based on participant report) are shown in Table 1. Slightly more than half of the participants $(53 \%)$ reported a family history of hearing loss. A history of tinnitus was reported by $39 \%$ of participants, and a history of head injury was reported by $28 \%$ of participants.

\section{Current Hearing Aids}

Three participants did not use any hearing aids. Those three individuals all had relatively better (although not normal) hearing in one ear (better-ear PTAs of 33,48 , and $65 \mathrm{~dB} \mathrm{HL}$ ). Among the 33 hearing aid users, $28(85 \%)$ were aided bilaterally. The remaining five participants $(15 \%)$ were aided unilaterally despite being candidates for bilateral amplification. Behind-theear aids were worn by $81 \%$ of the study participants and full-shell in-the-ear aids by $19 \%$. The participants had 
Table 1. Probable Etiologies of Study Participants, Based on Self-Reported History

\begin{tabular}{lcc}
\hline Probable Etiology & $\#$ & $\%$ \\
\hline Idiopathic & 2 & 5.6 \\
Congenital or genetic & 3 & 8.3 \\
Illness & 6 & 16.7 \\
Ototoxicity & 4 & 11.1 \\
$>1$ etiology & 6 & 16.7 \\
Aging & 15 & 41.7 \\
\hline
\end{tabular}

worn hearing aids, on average, for $19 \mathrm{yr}$ (range 2-67 yr). Average age of current hearing aids was $3.2 \mathrm{yr}$. Thus, in general, our participants were wearing aids of appropriate style for their hearing loss and were wearing aids of current technology.

The majority of our participants (87\%) wore hearing aids set to WDRC. The remaining participants (13\% of the sample) were using hearing aids set for linear processing. Individuals with poorer hearing thresholds were more likely to be wearing linear amplification $\left(t_{29}=2.21\right.$, $p=0.04)$. Mean PTA for the linear aid users was $79 \mathrm{~dB}$ HL, compared with $66 \mathrm{~dB}$ HL for the WDRC aid users. This may reflect the habit of some fitting audiologists to provide linear amplification to listeners with very poor hearing thresholds, or who previously used linear amplification (Keidser et al, 2007).

With regard to technology features, $50 \%$ of the hearing aids were set for omnidirectional processing, 30\% of the aids were set for automatic directivity, and $20 \%$ of the aids had a manual directional microphone. The mean reduction in gain when the directional mode was activated (calculated as average gain reduction at $0.5,1$, and $2 \mathrm{kHz}$ ) was $6 \mathrm{~dB}$ (As measured in the Verifit, that difference may have incorporated the effects of digital noise reduction, if present). Digital noise reduction was present in $73 \%$ of the hearing aids. The mean reduction in noise level was $4 \mathrm{~dB}$. Over half (53\%) of the hearing aid wearers did not have telecoils in their hearing aids or the telecoil had not been activated by their audiologist. Manual telecoils were used by $31 \%$ of the cohort and another $16 \%$ had automatically activated telecoils.

\section{Assistive Device Use and Aural Rehabilitation}

Considering the significant disability caused by severe loss and the expected benefit of assistive devices in difficult listening situations, the extent of assistive device use other than hearing aids was low. Only nine of 36 participants (25\%) reported any assistive device use. Of those, five participants (13\%) reported using a streamer to access their cell phone. One person used an amplified telephone. No person reported using a captioned phone. Three participants (8\%) had used remote microphones. Seven participants (19\%) reported having attended an aural rehabilitation class. Six of those seven participants were also assistive device users. A history of seeking treatment other than hearing aids (i.e., aural rehabilitation training or use of assistive devices) was not related to severity of loss, or to age ( $p>$ 0.05 in each case).

\section{Loudness Discomfort Levels and Dynamic Range}

Table 2 shows the mean and range values for loudness discomfort levels at the two test frequencies, as well as the corresponding dynamic ranges. Most listeners had significantly reduced dynamic ranges which precluded availability of the full range of speech cues (e.g., Zeng et al, 2002).

\section{Spectral Resolution}

For the spectral ripple test, there was no significant difference in threshold across the three test blocks $\left[F_{(2,46)}=1.48, p=0.24, \eta^{2}=0.06\right]$. Accordingly, the final threshold for each participant was calculated as the mean of the best two block scores. The mean ripple threshold was 1.5 ripples/octave ( $\mathrm{SD}=1.1$ ). For interpretation, the scores can be contrasted with those from Henry et al (2005). In that study, data from 32 listeners with mild to profound sensorineural hearing loss resulted in a mean score of 1.77 ripples/octave and a range from 0.33 to 4.97 ripples/octave (plotted as horizontal lines in Figure 2).

In general, there was no relationship between ripple threshold and amount of loss $(r=-0.13, p=0.50)$ (Figure 2). Of interest are two outliers with thresholds of approximately 5 ripples/octave, whose performance is $>2$ SDs above the current study group's mean (albeit within the range suggested by Henry et al's data). One was a 60 -yr-old male who reported a congenital loss. Despite relatively poor hearing (PTA $48.3 \mathrm{~dB}$ HL in the right ear and $76.7 \mathrm{~dB}$ HL in the left ear), he had never used hearing aids. The other outlier was a bilaterally aided 77-yr-old female who reported onset of loss at age $10 \mathrm{yr}$. In addition to early onset of hearing loss, both participants presented with a mixed loss.

\section{Dead Regions}

The TEN test could not be completed for some participants because of loudness discomfort (particularly at

Table 2. Range and Mean of Frequency-Specific Loudness Discomfort Levels (Averaged Across Ears)

\begin{tabular}{lccrr}
\hline & Minimum & Maximum & Mean & \multicolumn{1}{c}{ SD } \\
\hline LDL 0.5 kHz & 90.0 & 110.0 & 105.5 & 6.0 \\
LDL 2 kHz & 90.0 & 120.0 & 109.7 & 8.5 \\
Dynamic range $0.5 \mathrm{kHz}$ & 10.0 & 70.0 & 40.9 & 14.5 \\
Dynamic range $2 \mathrm{kHz}$ & 15.0 & 60.0 & 35.7 & 12.3 \\
\hline
\end{tabular}




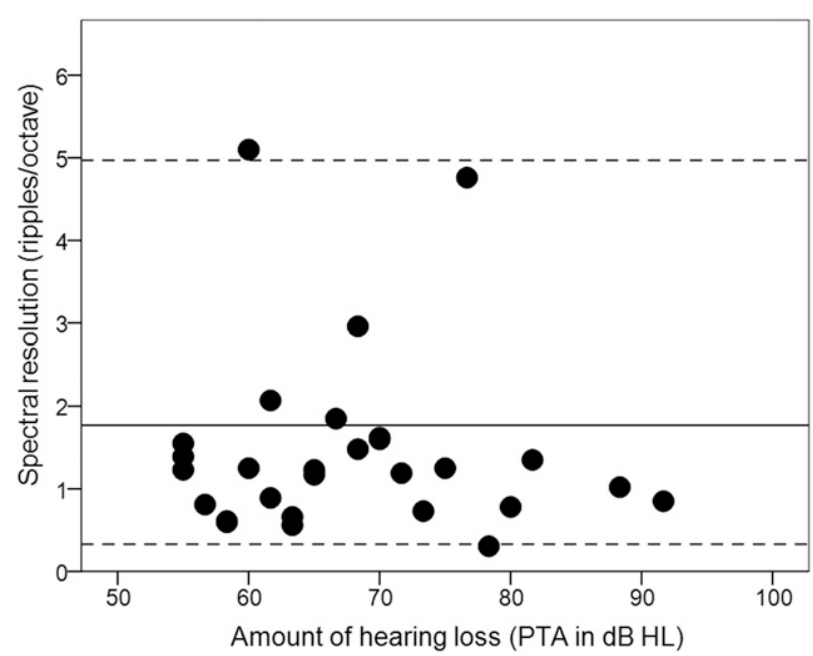

Figure 2. Spectral resolution (expressed as the final score on the spectral ripple test) as a function of amount of hearing loss (expressed as the three-frequency PTA). Higher ripple scores represent better performance. Horizontal lines show the mean (solid line) and score range (dashed lines) from Henry et al (2005).

$3 \mathrm{kHz}$ ), insufficient test time, or in one instance, an equipment problem. TEN data were available for 31 participants at $1 \mathrm{kHz}, 29$ participants at $2 \mathrm{kHz}$, and 26 participants at $3 \mathrm{kHz}$. Among those individuals, positive tests were noted for $10 \%$ of the participants at $1 \mathrm{kHz}, 21 \%$ of the participants at $2 \mathrm{kHz}$, and $15 \%$ of the participants at $3 \mathrm{kHz}$. There was a trend for listeners with poorer hearing to demonstrate more dead regions. The mean PTA was $66 \mathrm{~dB} \mathrm{HL}$ for those with negative TEN or a positive TEN at one frequency and $80 \mathrm{~dB}$ HL for those with positive TEN at two or three frequencies. However, there was no significant difference in PTA across those two groups $\left[F_{(23)}=1.33\right.$, $\left.p=0.29, \eta^{2}=0.12\right]$.

\section{Objective Speech Recognition}

The mean monosyllabic word score was $47.2 \%$ (SD = $24.7 \%$ ), calculated for 35 participants (For one participant, it was not possible to present the speech at suprathreshold levels without loudness discomfort). Figure 3 shows monosyllabic word recognition as a function of degree of loss (PTA). Unsurprisingly, listeners with more hearing loss had poorer word recognition. There was a significant negative correlation $(r=-0.70$ for the right ear and $r=-0.64$ for the left ear, $p<0.01$ in each case). However, there was also considerable variability, particularly for listeners with PTAs between 60 and $80 \mathrm{~dB}$ HL.

Mean QuickSIN was $17.5 \mathrm{~dB}(\mathrm{SD}=5.9 \mathrm{~dB})$. Figure 4 shows monaural QuickSIN data as a function of degree of loss (PTA). The variability was greatest for hearing losses between 60 and $70 \mathrm{~dB}$, with performance ranging from mild hearing in noise difficulty [i.e., signal-to-noise ratio (SNR) threshold between 4 and $7 \mathrm{~dB}$ ] to listeners who were unable to recognize speech even at the most favor-

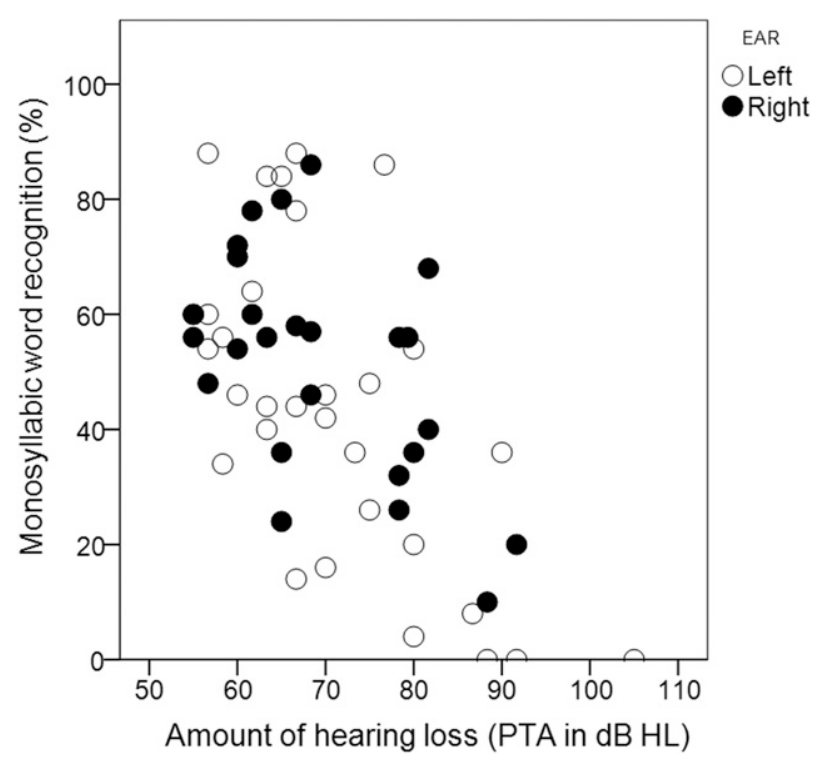

Figure 3. Monosyllabic word recognition as a function of amount of hearing loss (PTA). Higher word recognition scores represent better performance. Scores for right and left ears are shown by the filled and open circles, respectively.

able SNR available in the test. Listeners with more hearing loss had poorer speech-in-noise thresholds $(r=0.43$, $p<0.01$ ). Visually, the data suggest the following interpretation: listeners with $>70 \mathrm{~dB}$ of hearing loss will almost certainly have severe (Classification of speechin-noise ability according to the QuickSIN recommendations) speech-in-noise difficulty. Listeners with $<70 \mathrm{~dB}$ of hearing loss were approximately equally divided between moderate and severe speech-in-noise difficulty.

Figure 5 shows the relationship between monosyllabic word recognition and speech in noise thresholds. Overall, NU6 scores were strongly related to SNR thresholds $(r=$ -0.78 for the right ear and $r=-0.84$ for the left ear, $p<$ 0.01 in each case). Visually, the data suggest that for listeners with very poor monosyllabic word recognition (less than approximately 40\%), irrespective of their degree of hearing loss, the QuickSIN provides little additional information. Those listeners can be assumed to have severe speech-in-noise difficulty. Accurate quantification of speech-in-noise ability is most critical for listeners with monosyllabic word recognition of $40 \%$ or greater. As a group, those listeners demonstrated QuickSIN scores ranging from near-normal to severe.

\section{Contribution of Individual Factors to Speech Recognition}

To understand the sources of variability in speech recognition, regression models were used to analyze relationships between hearing loss (PTA), spectral resolution, and speech recognition.

Separate analyses were conducted for speech in quiet (NU6 word score) and speech in noise (QuickSIN threshold). 


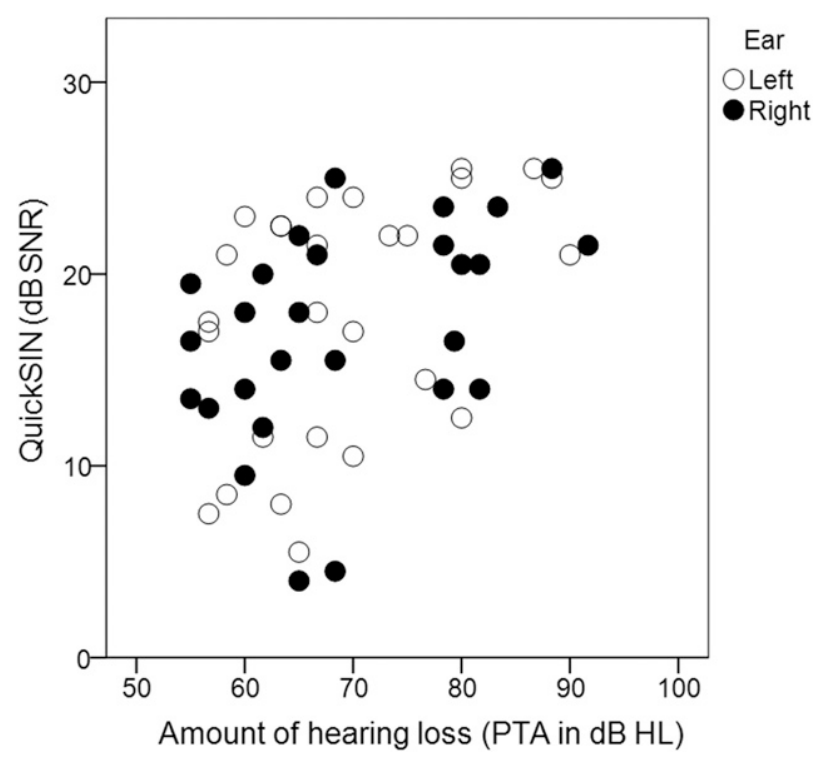

Figure 4. QuickSIN scores as a function of amount of hearing loss (PTA). Lower QuickSIN scores represent better performance. Scores for right and left ears are shown by the filled and open circles, respectively.

In each analysis, the independent variables were entered in four blocks: (a) amount of hearing loss (PTA in dB HL); (b) spectral resolution (ripple threshold in ripples/octave); (c) number of dead regions, assuming that more dead regions suggest more extensive cochlear damage; and (d) participant age (years). The regression analyses used an alpha-level criterion of 0.05 for probability of entry into the model and 0.1 for probability for removal from the model. The order of entry into the model was designed to examine the predictive variables according to largest

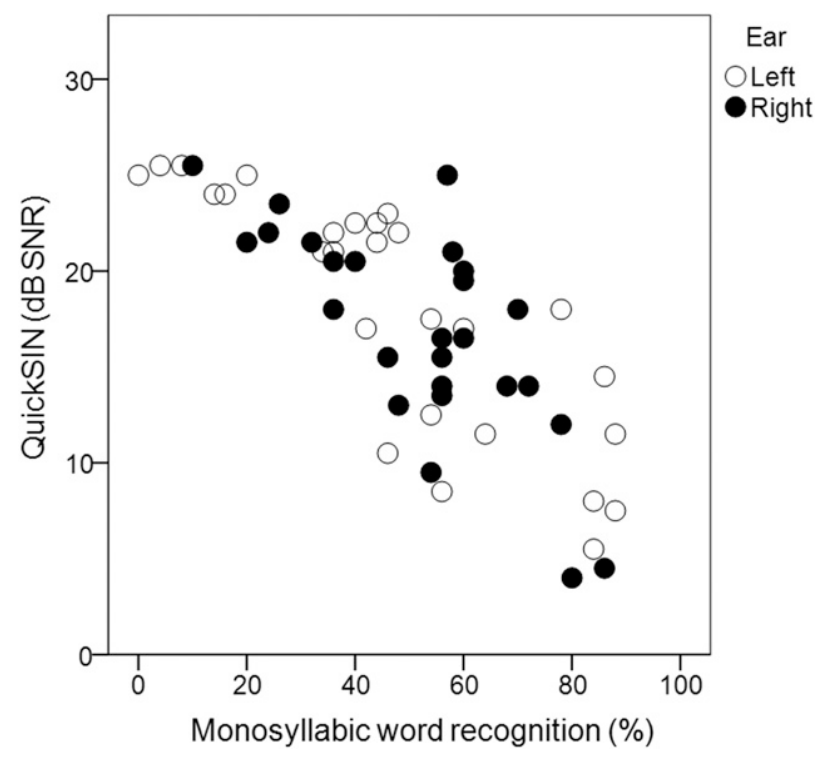

Figure 5. QuickSIN scores as a function of monosyllabic word recognition. Lower QuickSIN scores represent better performance. Scores for right and left ears are shown by the filled and open circles, respectively. hypothesized effects. Although not part of the study design, participant age was included in the models to account for any age-related variance. None of the independent variables was significantly correlated with another $(p>0.05$ in all cases), and collinearity within the models was acceptable (VIFs $<2.0$, tolerances $>0.5$ ) (Hair et al, 2010).

Results of the regression analysis for speech in quiet are shown in Table 3 . The dependent variable (percent correct NU6 words) was transformed to rationalize arcsine units to normalize variance across the score range (Studebaker, 1985). Where test data were unavailable, values were excluded listwise (Field, 2013), resulting in a set size of 28 participants. The initial model (Step 1) explained $23 \%$ of the variance $\left[F_{(1,26)}=7.58, p=0.011\right]$. Adding spectral resolution (Step 2) accounted for an additional $18 \%$ of the variance $\left[F_{(2,25)}=8.39, p=0.002\right]$. Adding the number of dead regions (Step 3) accounted for another $19 \%$ of the variance $\left[F_{(3,24)}=11.37, p<\right.$ 0.005]. Including age as a predictor did not account for significant amounts of additional variance. The final model accounted for $60 \%$ of the variance in NU6 score, with the variance approximately equally accounted for by PTA, spectral resolution, and number of dead regions.

Results of the regression analysis for speech in noise (QuickSIN threshold) are shown in Table 4. Where test data were unavailable, values were excluded listwise (Field, 2013), resulting in a set size of 27 participants. In the initial model (Step 1), PTA explained a modest $15 \%$ of the variance in QuickSIN threshold $\left[F_{(1,22)}=\right.$ $4.54, p=0.043]$. Adding additional factors did not improve the model fit.

\section{Subjective Perception of Communication}

For the SSQ questionnaire, 10\% of responses across all participants were given as "not applicable." In general, there was no pattern to those responses, although the highest numbers of "not applicable" responses (eight of 36 respondents) were in response to the questions about hearing a passenger while driving and whether, once visible, a sound source was further or closer than expected. The following analyses are based on all rated responses.

Previous work has shown that the subscale scores recommended by the test developers are appropriate for listeners with severe loss (Akeroyd et al, 2014). Mean subscale scores were 4.04 for the speech subscale, 4.41 for the spatial subscale, and 5.70 for the quality subscale. Although the three subscale scores were correlated with one another, there was no relationship between individual subscale scores and some of the possible contributors to those scores, including amount of hearing loss or speech recognition ability in noise (Table 5).

Figures 6-8 present a graphical representation of SSQ ratings (for the individual speech, spatial, and quality questions, respectively). For each question, the filled diamond shows the mean score for our cohort, 
Table 3. Summary of Regression Analysis for Variables Predicting Speech Recognition in Quiet

\begin{tabular}{|c|c|c|c|c|c|c|c|c|}
\hline & Variable & $\beta$ & $t$ & $R$ & $R^{2}$ & $\Delta R^{2}$ & $F$ & $p$ \\
\hline \multirow[t]{2}{*}{ Step 1} & & & & 0.48 & 0.23 & 0.23 & 7.58 & 0.01 \\
\hline & PTA & -0.48 & -2.75 & & & & & 0.01 \\
\hline \multirow[t]{3}{*}{ Step 2} & & & & 0.63 & 0.40 & 0.18 & 7.35 & 0.01 \\
\hline & PTA & -0.42 & -2.71 & & & & & 0.01 \\
\hline & Ripple & 0.42 & 2.71 & & & & & 0.01 \\
\hline \multirow[t]{4}{*}{ Step 3} & & & & 0.77 & 0.59 & 0.19 & 10.77 & $<0.01$ \\
\hline & PTA & -0.41 & -3.10 & & & & & 0.01 \\
\hline & Ripple & 0.40 & 3.00 & & & & & 0.01 \\
\hline & Dead regions & -0.43 & -3.28 & & & & & $<0.01$ \\
\hline \multirow[t]{5}{*}{ Step 4} & & & & 0.77 & 0.59 & 0.01 & 0.26 & 0.61 \\
\hline & PTA & -0.42 & -3.09 & & & & & 0.01 \\
\hline & Ripple & 0.38 & 2.81 & & & & & 0.01 \\
\hline & Dead regions & -0.42 & -3.08 & & & & & 0.01 \\
\hline & Age & -0.07 & -0.51 & & & & & 0.61 \\
\hline
\end{tabular}

Notes: $F$ and $p$ values in this table refer to the effect of adding additional variables. $F$ and $p$ values for the overall models are given in the text.

and error bars show $\pm 1 \mathrm{SD}$ about that mean. For each item, a rating of 0 represents poor perceived ability, and a rating of 10 represents good perceived ability. For comparison, mean scores are also shown for listeners with mild-to-moderate loss, as reported by Noble and Gatehouse (2006). Downward pointing triangles show reported means for unaided listeners with mild-to-moderate loss, and upward pointing triangles show reported means for bilaterally aided listeners with mild-to-moderate loss. Those comparisons are considered further in the discussion.

Consistent with the poor measured speech-in-noise in the present cohort, ratings that addressed the ability to follow a talker in a background of any other signal were generally low. The only highly rated speech situation was the ability to follow a single talker in a quiet, carpeted room (mean rating $=7.4$ ). Next highest mean ratings were given for the two other questions which addressed speech in quiet: one talker at a time in a group in quiet; and the ability to follow a phone conversation (mean ratings $=5.8$ ). The third highest mean rating was given for ability to follow a single talker in a background of low level, nonspeech noise such as a fan (mean rating $=5.6$ ). All other speech scenarios were rated $<5$. Particularly low ratings were given to scenarios which required two simultaneous targets (e.g., talker plus telephone or television).

Somewhat surprisingly, because most of participants had symmetrical hearing loss, many of the spatial questions regarding location or direction of sounds indicated low perceived ability. In some cases, this may be related to impaired audibility, such as the ability to tell how far away something was (i.e., intensity is a cue for proximity).

Quality ratings were moderately high. Participants felt, on average, that other voices, everyday sounds, and their own voice sounded natural. Music quality was rated positively, and they were able to distinguish different musical instruments. However, low ratings were given to

Table 4. Summary of Regression Analysis for Variables Predicting Speech Recognition in Noise

\begin{tabular}{|c|c|c|c|c|c|c|c|c|}
\hline & Variable & $\beta$ & $t$ & $R$ & $R^{2}$ & $\Delta R^{2}$ & $F$ & $p$ \\
\hline \multirow[t]{2}{*}{ Step 1} & & & & 0.39 & 0.15 & 0.15 & 4.54 & 0.04 \\
\hline & PTA & 0.39 & 2.13 & & & & & 0.04 \\
\hline \multirow[t]{3}{*}{ Step 2} & & & & 0.46 & 0.22 & 0.06 & 1.87 & 0.19 \\
\hline & PTA & 0.36 & 1.99 & & & & & 0.06 \\
\hline & Ripple & -0.25 & -1.37 & & & & & 0.19 \\
\hline \multirow[t]{4}{*}{ Step 3} & & & & 0.52 & 0.27 & 0.06 & 1.88 & 0.18 \\
\hline & PTA & 0.36 & 2.03 & & & & & 0.05 \\
\hline & Ripple & -0.24 & -1.33 & & & & & 0.20 \\
\hline & $\begin{array}{l}\text { Dead } \\
\text { regions }\end{array}$ & 0.24 & 1.37 & & & & & 0.18 \\
\hline \multirow[t]{5}{*}{ Step 4} & & & & 0.52 & 0.27 & $<0.01$ & 0.01 & 0.92 \\
\hline & PTA & 0.37 & 1.99 & & & & & 0.06 \\
\hline & Ripple & -0.24 & -1.26 & & & & & 0.22 \\
\hline & $\begin{array}{l}\text { Dead } \\
\text { regions }\end{array}$ & 0.24 & 1.32 & & & & & 0.20 \\
\hline & Age & 0.02 & 0.10 & & & & & 0.92 \\
\hline
\end{tabular}

Notes: $F$ and $p$ values in this table refer to the effect of adding additional variables. $F$ and $p$ values for the overall models are given in the text. 
Table 5. Pearson's Correlation Coefficients between Rated Ability Measured by SSQ Subscores and Possible Predictive Factors

\begin{tabular}{|c|c|c|c|c|c|}
\hline & $\begin{array}{c}\text { PTA } \\
\text { Better Ear }\end{array}$ & QuickSIN AU & SSQ Speech & SSQ Quality & SSQ Spatial \\
\hline PTA better ear & 1 & $0.536^{\star \star}$ & -0.212 & -0.241 & -0.110 \\
\hline QuickSIN AU & $0.536^{\star \star}$ & 1 & -0.328 & -0.231 & 0.017 \\
\hline SSQ speech & -0.212 & -0.328 & 1 & $0.668^{\star \star}$ & $0.557^{\star *}$ \\
\hline SSQ quality & -0.241 & -0.231 & $0.668^{\star *}$ & 1 & $0.847^{\star *}$ \\
\hline SSQ spatial & -0.110 & 0.017 & $0.557^{\star \star}$ & $0.847^{\star \star}$ & 1 \\
\hline
\end{tabular}

Note: ${ }^{*}$ Significant at $p<0.05$.

those aspects of the quality subscale which addressed speech-in-noise listening, such as while driving. The ratings also acknowledged the high level of effort and concentration required while listening.

\section{DISCUSSION}

$\mathrm{T}$ he large variability in speech recognition has long been of interest to researchers and clinicians. Many studies have attempted to identify contributors to this variability, particularly for listeners with mild-to-moderate loss. The present dataset focuses on these issues in a sample of listeners with severe loss (defined here as PTA $>55 \mathrm{~dB}$ HL). The following sections consider the implications of the relationships shown in the present data.

\section{Individual Factors that Contribute to Speech Recognition in Quiet}

The present data indicate a large variability in performance, particularly for participants with hearing loss (expressed as PTA) between 60 and $80 \mathrm{~dB}$ HL. Hierarchical linear regression models indicated that for words in quiet, PTA, spectral resolution (spectral ripple threshold), and number of dead regions were all associated with speech recognition. Interestingly, those three predictors were all similarly strong, and measures of spectral resolution and the number of dead regions accounted for nearly as much variance as the amount of hearing loss. Moreover, both spectral resolution and number of dead regions accounted for additional variance after audibility (e.g., PTA) had been accounted for. To summarize that finding in another way, it is not simply that the more hearing loss you have, the poorer your spectral resolution. Rather, for a certain level of loss, poorer spectral resolution means relatively poorer word recognition and better spectral resolution means relatively better word recognition.

This finding makes sense if we reflect on what is required for monosyllabic word recognition. With no contextual or visual cues, the listener must depend on audibility and discrimination of phonemes. Regarding audibility, a listener is unlikely to identify a consonant that is not sufficiently audible. Although in our protocol the words were presented at suprathreshold levels relative to PTA, as is done clinically, that method does not ensure audibility of the full bandwidth particularly for higher-frequency consonants.

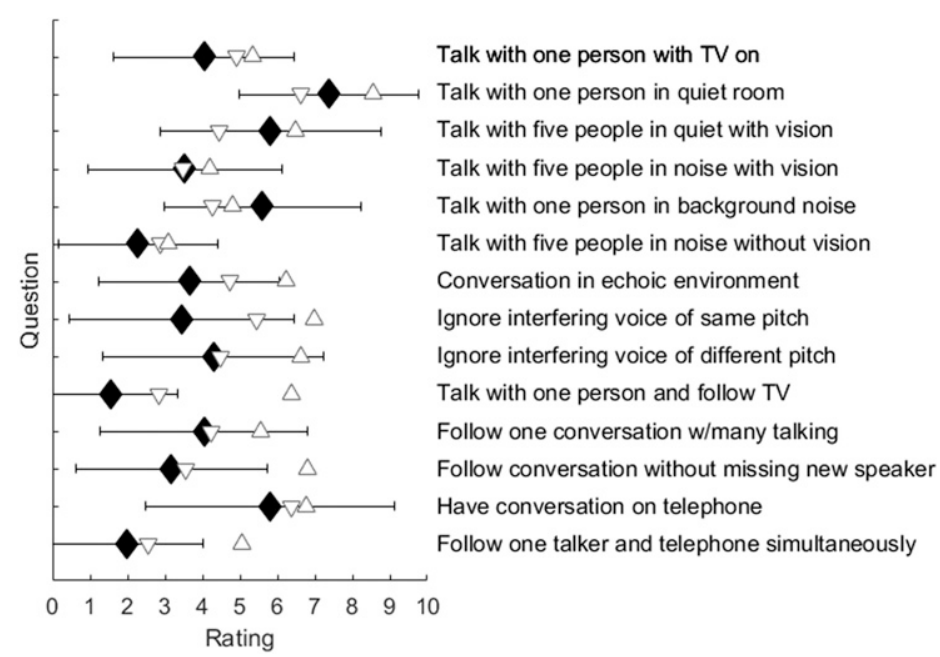

Figure 6. Subjective ratings for the 14 speech questions from the SSQ. Mean ratings are shown by the filled diamonds. Error bars show \pm 1 SD about the mean. Higher SSQ ratings represent better perceived ability. For comparison, mean scores are shown for listeners with mild-to-moderate hearing loss evaluated by Noble and Gatehouse (2006). Downward pointing triangles show the Noble and Gatehouse mean score for unaided listeners and upward pointing triangles show mean score for aided listeners. 


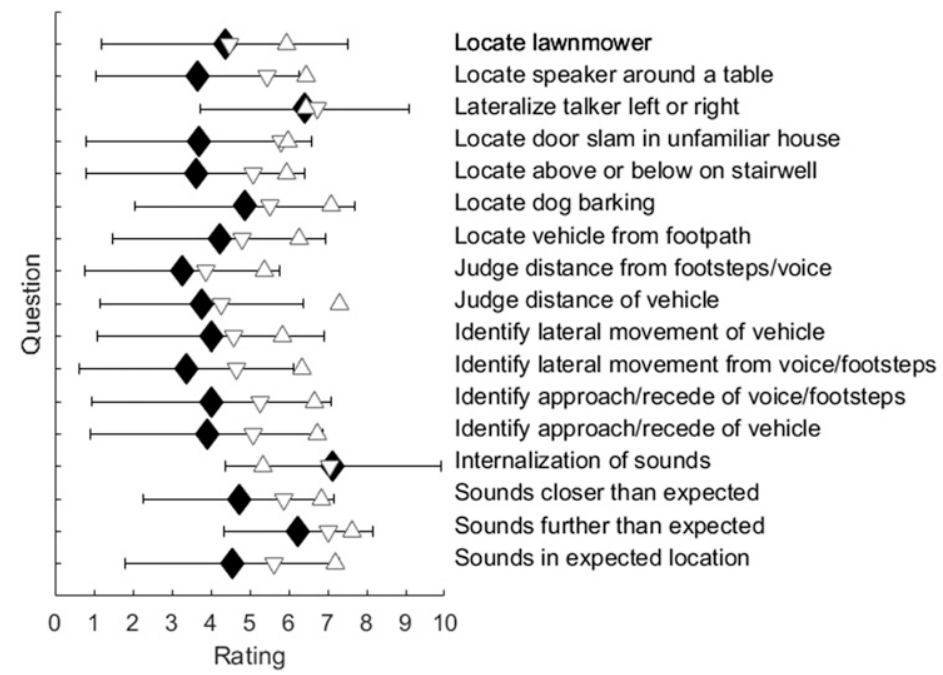

Figure 7. As in Figure 6, but for the 17 spatial questions from the SSQ.

The additional variance explained by spectral resolution suggests that even speech with adequate audibility is poorly discriminated by listeners with poor spectral resolution, quantified here as spectral ripple thresholds. Identification of consonant place is particularly dependent on adequate spectral resolution, because place is distinguished by unique information in the spectral domain (spectral shape and/or formant transition) (e.g., Shamma, 1988; Hedrick and Younger, 2001). When spectral information is severely degraded (as in vocoding studies), the ability to identify place is severely limited (e.g., Turner et al, 1995). Spectral resolution is also important for vowel identification and for accurate representation of consonant-vowel transitions. Moreover, the significant contribution of spectral resolution to variability in speech recognition reinforces that listeners with severe loss vary in spectral resolution, rather than presenting a homogeneously poor performance.

When dead regions are present, any consonant information obtained via off-frequency listening is less likely to be interpretable (e.g., Won et al, 2015). The proportion of our test participants with positive TEN tests was lower than in other studies with similar audiograms (Moore et al, 2003; Preminger et al, 2005; Ahadi et al, 2015). However, previous work found dead regions to be more likely at higher frequencies, where the cochlea is more vulnerable to damage and, also, somewhat more likely in cases of sloping hearing loss. Thus, discrepancies with some earlier work may reflect both participants' audiometric configuration and the choice of test frequencies.

In this study, we categorized the results of the TEN test according to the number of positive results. Note

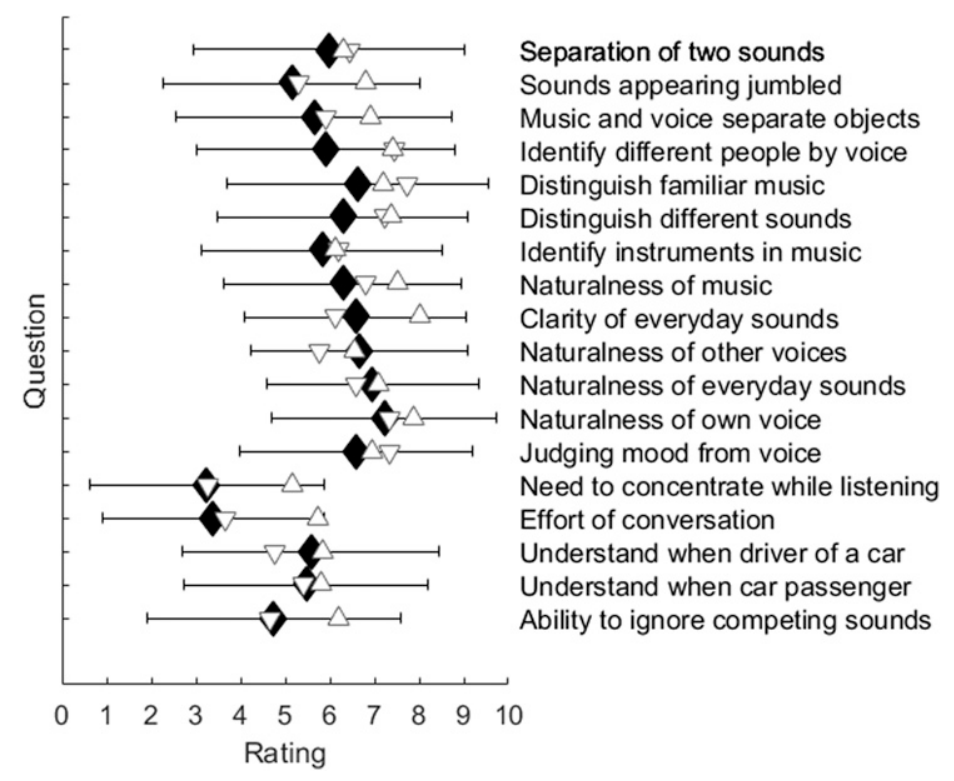

Figure 8. As in Figure 6, but for the 18 sound quality questions from the SSQ. 
that this categorization does not comprehensively assess the extent of dead regions, because the test was conducted only at specific frequencies. Indeed, the TEN itself is an indirect assessment of the likely presence of a dead region. Nonetheless, having a higher number of positive TEN tests suggests a more widespread cochlear damage pattern.

If spectral resolution and dead regions are related to speech recognition, should we be measuring these abilities in the clinic? Clinical versions of the tests are available (Moore et al, 2004; Drennan et al, 2014). Ching and colleagues (Ching et al, 2011; Ching and Dillon, 2013;) recently noted that tests of spectral resolution and dead regions did not account for additional variance in performance once pure-tone threshold had been accounted for. Although Ching and Dillon's findings appear to contradict the present study, they were based on a wider range of hearing thresholds. Because listeners with less hearing loss are expected to demonstrate more homogenous (and better) spectral resolution, it may be that specific measures of spectral resolution are valuable only in a more impaired population. Ching and colleagues also used a different measure of spectral resolution (psychoacoustic tuning curves), albeit one which is related to ripple thresholds (Davies-Venn et al, 2015).

Regarding dead regions, whereas some articles have supported the use of the TEN in clinical decision-making (e.g.,Mackersie et al, 2004; Moore, 2004; Preminger et al, 2005), others have suggested that it provides little additional information (e.g., Cox et al, 2012). In particular, listeners with and without high-frequency dead regions perform similarly with high-frequency amplification (Cox et al, 2011; 2012). To date, those studies have focused on listeners with sloping loss rather than the relatively flat losses of the present study. Because listeners with sloping loss would be expected to have relatively better low-to-mid frequency resolution, high-frequency dead regions may have a smaller impact on overall speech recognition. It is possible that when dead regions occur in the midfrequency range (as here, where as many as $20 \%$ of the sample had positive TEN), there will be a greater rehabilitation impact.

\section{Individual Factors that Contribute to Speech Recognition in Noise}

With regard to speech in noise, only a modest proportion of performance variance was explained by audibility (PTA). That finding is consistent with the implementation of the QuickSIN, in which overall audibility was set by patient perception ("loud but ok") and audibility of the target speech is more controlled by the sentence SNR than by presentation level. The suprathreshold measures (spectral resolution or dead regions) did not contribute significantly to QuickSIN score. The lack of relationship between speech in noise and spectral resolution is somewhat surprising, given the assumption that listeners with broader auditory filters will be more susceptible to energetic masking. Perhaps the QuickSIN stimuli-which mimic a realistic listening scenario with multiple background talkers-rely more heavily on other abilities not measured here, such as ability to listen in gaps (i.e., temporal resolution) and/or higher-order processing to focus on the target speech and inhibit other auditory streams.

Bernstein and colleagues (Bernstein et al, 2013; Mehraei et al, 2014) have recently provided another perspective on the importance of spectral resolution for speech understanding, through the use of spectrotemporal modulation (STM) detection. A model that included both STM thresholds and audibility was able to account for over $80 \%$ of the variance in speech understanding in broadband noise. STM is a signal that was first developed as a way of characterizing the receptive fields of auditory cortical neurons (Shamma, 2001). For this reason, the relationships with speech perception have been taken as further evidence that STM in some way captures a fundamental building block of auditory representations at the cortical level (Elhilali et al, 2003). It may be that the importance of spectral resolution observed in this study is related to the importance of STM, but that sensitivity to the slow temporal modulation of spectral information (around $4 \mathrm{~Hz}$ ) is a closer approximation to the information needed to understand speech. This may also explain why, in the current study, the spectral resolution thresholds predicted speech in quiet but not speech in noise, where tracking slowly varying spectrotemporal information may be essential to tracking one voice among competing voices or noise.

\section{Subjective Speech Perception and Quality}

The SSQ data in Figures 6-8 present an interesting qualitative comparison with previous work. The comparison data by Noble and Gatehouse (2006) represent 144 older adults (mean age $=68 \mathrm{yr}$ ) with mild-to-moderate loss who did not wear hearing aids (downward pointing triangles in figures) and 42 older adults (mean age = $66 \mathrm{yr}$ ) with mild-to-moderate loss who wore bilateral hearing aids (upward pointing triangles in figures). In general, for the speech and spatial questions, our sample of listeners with severe loss, despite wearing appropriate amplification, are more similar to listeners with mild-tomoderate loss who do not use hearing aids than to hearing aid users. In particular, bilaterally aided mild-to-moderate listeners rated their abilities higher than did our cohort for situations that required the listener to switch among multiple speakers or follow simultaneous auditory streams. For the quality questions, our cohort rated themselves similarly to mild-to-moderate listeners both with and without hearing aids. The exception were the questions which asked about effort and concentration, where aided 
listeners with mild-to-moderate loss rated themselves higher than either of the other groups.

We found no relationship between SSQ ratings and the objective predictors likely to underlie those ratings, such as audibility (PTA) or objective speech scores in quiet or in noise. This may reflect the subjective nature of the SSQ. To put this another way, the SSQ may reflect a listener's reaction to a communication difficulty more globally than it can capture the specific sources of that difficulty. Work on other populations has shown similar weak relationships between SSQ ratings and objective predictors (e.g., Stansell et al, 2015).

Note that the SSQ was administered here as a paperand-pencil questionnaire, rather than via interview questions. Although the interview approach was recommended at the time of test development (Gatehouse and Noble, 2004), the questionnaire approach has subsequently become common. The questionnaire approach has been noted to have lower test-retest reliability (Singh and Pichora-Fuller, 2010), but also avoids intentional bias by the interviewer; for example, if a question is expanded upon or explained. In the case of the present data, the respondent completed the questionnaire in a quiet test room. The tester reviewed any blank responses to ensure that the participant intended a "not applicable" response and clarified confusion about the response method as needed. Thus, our approach is likely to be more reliable than completion of the questionnaire under unknown conditions (i.e., distributed and returned by mail).

\section{Hearing Loss}

With regard to type of hearing loss, study inclusion criteria allowed individuals with air-bone gaps exceeding $10 \mathrm{~dB}$ to participate, with the rationale that individuals with severe hearing loss whose loss included a conductive component would experience many of the same communication difficulties as severe sensorineural hearing loss. Inclusion of those individuals was a deliberate choice to represent patients who might present with severe loss in clinical practice. The cohort included four such individuals. One had a history of tympanic membrane perforation, one had a history of cholesteatoma, and two had lowfrequency air-bone gaps of unknown etiology. In all four cases, bone-conduction thresholds indicated substantial cochlear pathology. With regard to test performance, two of these participants were outliers on the spectral resolution test, with relatively good scores of approximately five ripples per octave. However, it was not clear that the better performance was related to having a mixed loss. Henry et al (2005) reported similarly high scores from a participant with severe sensorineural loss. Moreover, the other two mixed-loss participants' scores were close to the mean score for the sensorineural loss participants. Finally, none of the mixed-loss participants were outliers on any other measure, including the speechrecognition tests. In general, these data suggest that individuals with severe mixed loss are likely to experience many or all of the same communication difficulties as individuals with severe sensorineural loss.

There was some suggestion in our data-as has been the case in other studies (e.g., Seldran et al, 2011)—that older adults who acquired severe loss later in life had more difficulty compensating for the loss than those earlyimpaired listeners. Specifically, the listeners who reported onset of loss before $20 \mathrm{yr}$ of age had better speech recognition in quiet (66\% compared to $48 \%$ for those with later onset of loss) and in noise (QuickSIN $9 \mathrm{~dB}$ compared with $14 \mathrm{~dB}$ for those with later onset of loss), despite having similar degrees of loss (PTA $72 \mathrm{~dB} \mathrm{HL}$, compared with $68 \mathrm{~dB}$ HL for those with later onset of loss). This may be related to a window of cortical plasticity following sensory damage (Glick and Sharma, 2017). However, it may also be associated with the fact that our early-impaired participants had quite different etiology (typically congenital loss or loss onset following an acute illness) than the participants with acquired loss. Although the size of the present sample was not sufficient to fully test these effects, such comparisons present an interesting possibility for future study.

\section{Hearing Technology}

The majority of our participants were wearing behindthe-ear hearing aids that were $<5$ years old, and were thus broadly appropriate for individuals with severe loss. In addition to the higher gain and output possible with behind-the-ear hearing aids, behind-the-ear aids can be ordered with telecoils and direct audio input, both potentially useful features for severe loss. However, half of the participants were wearing omnidirectional microphones, including some individuals with very poor speech-innoise scores. Among the hearing aids with active directional microphones, some provided relatively poor directionality in our electroacoustic tests. Finally, for those users with manual directional microphones, many individuals could not describe appropriate use of the directional setting. A common experience was that the participant referred to the directional setting as a "restaurant setting" but was unable to describe proper positioning (i.e., positioning oneself in such a way as to spatially separate the target talker from the noise thus maximizing directional benefit).

Only three participants reported having used hearing assistive technologies with remote microphones. This is unfortunate given the poor objective and subjective speech-in-noise recognition in this group and the limited capabilities of hearing aids (even with directional microphones) to improve communication in adverse listening environments. Remote microphones have been shown to be of very high benefit, improving speech recognition and 
reducing listening effort in noisy environments (e.g., Wolfe et al, 2015). We strongly encourage patient education in this regard. A demonstration system (such as an FM system with neck loop accessed via the patient's manual telecoil) provides a compelling listening experience and takes only a few minutes to demonstrate in a (noisy) sound field environment.

At the conclusion of the study, several of our participants were referred to their dispensing audiologist for activation of their hearing aid telecoil to allow loop access. We also referred two participants for cochlear implant evaluations (one of whom subsequently obtained an implant) and assisted two participants in obtaining captioned phones. In all of those cases, the participant indicated they were unaware that such solutions existed.

\section{Clinical Implications}

The present data suggest that the range of abilities of an individual can be more fully captured with comprehensive testing, supplementing the pure-tone testing and monosyllables in quiet which are most often used clinically. In particular, the listeners' ability to recognize speech in quiet was linked to spectral resolution and to the presence of dead regions. We propose that a valuable aspect of additional tests for listeners with severe loss is the opportunity for informed counseling. Many hearing aid wearers, including many of the participants in the present study, are focused on the capabilities of their hearing aids. They tend to attribute speech-recognition failures to device failure, with little to no recognition that perception requires suprathreshold processing unique to the individual. This is a particular problem for listeners with severe loss who are very likely to have suboptimal hearing aid experiences.

Individual counseling which offers concrete information about the status of the patient's auditory system (i.e., a spectral resolution score) can calibrate expectations for the hearing aid and lead to greater acceptance of complementary auditory treatment. We found such counseling to have a very positive effect. Many of our participants commented that no provider had ever discussed the contributions to their communication difficulties, or the extent to which hearing aid use would or would not address those issues. However, both the TEN and ripple tests must be weighed against other needs in the context of a limited appointment time.

Overall, we found our sample to be greatly underserved in terms of solutions other than hearing aids. We recognize that this may reflect self-selection, in that our participants responded to advertisements focused on speech recognition in severe loss. However, we believe it may also reflect some dispensers' lack of familiarity with assistive devices other than those devices most commonly supported by manufacturers' training (such as phone streamers). We strongly encourage providing information to patients regarding nonhearing aid options, such as hearing assistive technology, cochlear implant candidacy (if appropriate), aural rehabilitation, and auditory training. Handouts, brief counseling, or referral to a local Hearing Loss Association of American group are relatively low-effort strategies in terms of time spent but likely to result in improved objective and perceived ability in problematic situations (e.g., noise and reverberation) as well as more realistic expectations for those situations. Such information also reinforces audiologists' role as a comprehensive resource for communication issues beyond those addressed by hearing aids.

Acknowledgments. The authors thank Arleen Li, Laura Mathews, and Amanda Mueller for their assistance with study data.

\section{REFERENCES}

Ahadi M, Milani M, Malayeri S. (2015) Prevalence of cochlear dead regions in moderate to severe sensorineural hearing impaired children. Int J Pediatr Otorhinolaryngol 79(8):1362-1365.

Akeroyd MA, Guy FH, Harrison DL, Suller SL. (2014) A factor analysis of the SSQ (speech, spatial, and qualities of hearing scale). Int $J$ Audiol 53(2):101-114.

Audioscan. (2016) Audioscan Verifit User's Guide 3.16. Dorchester, ON, Canada: Audioscan.

Bernstein JG, Mehraei G, Shamma S, Gallun FJ, Theodoroff SM, Leek MR. (2013) Spectrotemporal modulation sensitivity as a predictor of speech intelligibility for hearing-impaired listeners. J Am Acad Audiol 24(4):293-306.

Boothroyd A, Springer N, Smith L, Schulman J. (1988) Amplitude compression and profound hearing loss. J Speech Hear Res 31(3): 362-376

Byrne D, Dillon H, Ching T, Katsch R, Keidser G. (2001) NAL-NL1 procedure for fitting nonlinear hearing aids: characteristics and comparisons with other procedures. J Am Acad Audiol 12(1): 37-51.

Centers for Disease Control and Prevention (CDC). (2011) Severe hearing impairment among military veterans-United States, 2010. MMWR Morb Mortal Wkly Rep 60(28):955-958.

Cheatham MA, Dallos P. (2000) The dynamic range of inner hair cell and organ of Corti responses. J Acoust Soc Am 107(3):1508-1520.

Ching TY, Dillon H. (2013) A brief overview of factors affecting speech intelligibility of people with hearing loss: implications for amplification. Am J Audiol 22(2):306-309.

Ching TY, Dillon H, Lockhart F, van Wanrooy E, Flax M. (2011) Audibility and speech intelligibility revisited: implications for amplification. In: Dau T, Jepsen ML, Poulsen T, eds. Speech Perception and Auditory Disorders: 3rd International Symposium on Auditory and Audiological Research. Denmark: The Danavox Jubilee Foundation, pp. 11-19.

Cole B. (2009) Verifit \& RM500SL Test Signals \& Analysis Audionote 2. Windsor, ON: Etymonic Design Incorporated.

Convery E, Keidser G. (2011) Transitioning hearing aid users with severe and profound loss to a new gain/frequency response: benefit, perception, and acceptance. J Am Acad Audiol 22(3): 168-180. 
Cox RM, Alexander GC, Johnson J, Rivera I. (2011) Cochlear dead regions in typical hearing aid candidates: prevalence and implications for use of high-frequency speech cues. Ear Hear 32(3): $339-348$.

Cox RM, Alexander GC, Taylor IM, Gray GA. (1997) The contour test of loudness perception. Ear Hear 18(5):388-400.

Cox RM, Johnson JA, Alexander GC. (2012) Implications of highfrequency cochlear dead regions for fitting hearing aids to adults with mild to moderately severe hearing loss. Ear Hear 33(5): $573-587$.

Cruickshanks KJ, Wiley TL, Tweed TS, Klein BE, Klein R, MaresPerlman JA, Nondahl DM. (1998) Prevalence of hearing loss in older adults in Beaver Dam, Wisconsin. The Epidemiology of Hearing Loss Study. Am J Epidemiol 148(9):879-886.

Davies-Venn E, Nelson P, Souza P. (2015) Comparing auditory filter bandwidths, spectral ripple modulation detection, spectral ripple discrimination, and speech recognition: normal and impaired hearing. J Acoust Soc Am 138(1):492-503.

Davies-Venn E, Souza P. (2014) The role of spectral resolution, working memory, and audibility in explaining variance in susceptibility to temporal envelope distortion. J Am Acad Audiol 25(6): 592-604

Davies-Venn E, Souza P, Brennan M, Stecker GC. (2009) Effects of audibility and multichannel wide dynamic range compression on consonant recognition for listeners with severe hearing loss. Ear Hear 30(5):494-504

Dillon H. (1988) Compression in hearing aids. In: Sandlin R, ed. Handbook of Hearing Aid Amplification Volume I: Theoretical and Technical Considerations. New York: College-Hill Press.

Drennan WR, Anderson ES, Won JH, Rubinstein JT. (2014) Validation of a clinical assessment of spectral-ripple resolution for cochlear implant users. Ear Hear 35(3):e92-e98.

Elhilali M, Chi T, Shamma S. (2003) A spectro-termporal modulation index (STMI) for assessment of speec intelligibility. Speech Commun 41:331-348.

Field A. (2013) Discovering Statistics Using IBM SPSS Statistics. 4th ed. London: Sage Publications Ltd.

Flynn MC, Dowell RC, Clark GM. (1998) Aided speech recognition abilities of adults with a severe or severe-to-profound hearing loss. $J$ Speech Lang Hear Res 41(2):285-299.

Gatehouse S, Noble W. (2004) The speech, spatial and qualities of hearing scale (SSQ). Int J Audiol 43(2):85-99.

Gevonden MJ, Myin-Germeys I, van den Brink W, van Os J, Selten JP, Booij J. (2015) Psychotic reactions to daily life stress and dopamine function in people with severe hearing impairment. Psychol Med 45(8):1665-1674.

Glick H, Sharma A. (2017) Cross-modal plasticity in developmental and age-related hearing loss: clinical implications. Hear Res 343:191-201.

Hair JFJ, Black WC, Babin BJ, Anderson RE. (2010) Multivariate Data Analysis. Upper Saddle River, NJ: Prentice Hall.

Hamernik RP, Patterson JH, Turrentine GA, Ahroon WA. (1989) The quantitative relation between sensory cell loss and hearing thresholds. Hear Res 38(3):199-211.

Hawkins DB, Walden BE, Montgomery A, Prosek RA. (1987) Description and validation of an LDL procedure designed to select SSPL90. Ear Hear 8(3):162-169.
Hedrick M, Younger MS. (2001) Perceptual weighting of relative amplitude and formant transition cues in aided CV syllables. $J$ Speech Lang Hear Res 44(5):964-974.

Henry BA, Turner CW, Behrens A. (2005) Spectral peak resolution and speech recognition in quiet: normal hearing, hearing impaired, and cochlear implant listeners. J Acoust Soc Am 118(2): $1111-1121$

Humes LE. (2007) The contributions of audibility and cognitive factors to the benefit provided by amplified speech to older adults. J Am Acad Audiol 18(7):590-603.

Keidser G, Dillon H, Dyrlund O, Carter L, Hartley D. (2007) Preferred low- and high-frequency compression ratios among hearing aid users with moderately severe to profound hearing loss. $J \mathrm{Am}$ Acad Audiol 18(1):17-33.

Killion MC, Niquette PA, Gudmundsen GI, Revit LJ, Banerjee S. (2004) Development of a quick speech-in-noise test for measuring signal-to-noise ratio loss in normal-hearing and hearing-impaired listeners. J Acoust Soc Am 116(4 Pt 1):2395-2405.

Kochkin S. (2009) MarkeTrak VIII: 25-year trends in the hearing health market. Hear Rev 16:12-31.

Kochkin S. (2011) MarkeTrak VIII: mini-BTEs tap new market, users more satisfied. Hear $J$ 64:17-24.

Kühnel V, Margolf-Hackl S, Kiessling J. (2001) Multi-microphone technology for severe-to-profound hearing loss. Scand Audiol Suppl 30(52):65-68.

Lyxell B, Andersson U, Borg E, Ohlsson I-S. (2003) Workingmemory capacity and phonological processing in deafened adults and individuals with a severe hearing impairment. Int J Audiol 42 (Suppl 1):S86-S89.

Mackersie CL, Crocker TL, Davis RA. (2004) Limiting highfrequency hearing aid gain in listeners with and without suspected cochlear dead regions. J Am Acad Audiol 15(7):498-507.

Mathworks. (2014) MATLAB. Natick, MA.

McArdle RA, Wilson RH. (2006) Homogeneity of the 18 QuickSIN lists. J Am Acad Audiol 17(3):157-167.

Mehraei G, Gallun FJ, Leek MR, Bernstein JG. (2014) Spectrotemporal modulation sensitivity for hearing-impaired listeners: dependence on carrier center frequency and the relationship to speech intelligibility. J Acoust Soc Am 136(1):301-316.

Moodie S, Scollie S, Seewald R, Bagatto M, Beaulac S. (2007) The DSL method for pediatric and adult hearing instrument fitting: Version 5 Phonak Focus. Vol. 37. Stäfa, Switzerland: Phonak AG.

Moore BC. (2004) Dead regions in the cochlea: conceptual foundations, diagnosis, and clinical applications. Ear Hear 25(2): 98-116.

Moore BC. (2010) Testing for cochlear dead regions: audiometer implementation of the TEN(HL) test. Hear Rev 17:10-16.

Moore BC, Glasberg BR, Stone MA. (2004) New version of the TEN test with calibrations in dB HL. Ear Hear 25(5):478-487.

Moore BC, Killen T, Munro KJ. (2003) Application of the TEN test to hearing-impaired teenagers with severe-to-profound hearing loss. Int J Audiol 42(8):465-474.

Noble W, Gatehouse S. (2006) Effects of bilateral versus unilateral hearing aid fitting on abilities measured by the speech, spatial, and qualities of hearing scale (SSQ). Int $J$ Audiol 45(3):172-181. 
Preminger JE, Carpenter R, Ziegler CH. (2005) A clinical perspective on cochlear dead regions: intelligibility of speech and subjective hearing aid benefit. J Am Acad Audiol 16(8):600-613. quiz 631-632.

Ryan A, Dallos P. (1975) Effect of absence of cochlear outer hair cells on behavioural auditory threshold. Nature 253(5486):44-46.

Seldran F, Gallego S, Micheyl C, Veuillet E, Truy E, Thai-Van H. (2011) Relationship between age of hearing-loss onset, hearingloss duration, and speech recognition in individuals with severeto-profound high-frequency hearing loss. J Assoc Res Otolaryngol 12(4):519-534.

Shamma S. (2001) On the role of space and time in auditory processing. Trends Cogn Sci 5(8):340-348.

Shamma SA. (1988) The acoustic features of speech sounds in a model of auditory processing: vowels and voiceless fricatives. J Phonetics 16:77-91.

Singh G, Pichora-Fuller MK. (2010) Older adults performance on the speech, spatial, and qualities of hearing scale (SSQ): test-retest reliability and a comparison of interview and self-administration methods. Int J Audiol 49(10):733-740.

Souza PE, Bishop RD. (1999) Improving speech audibility with wide dynamic range compression in listeners with severe sensorineural loss. Ear Hear 20(6):461-470.

Souza PE, Boike KT, Witherell K, Tremblay K. (2007) Prediction of speech recognition from audibility in older listeners with hearing loss: effects of age, amplification, and background noise. J Am Acad Audiol 18(1):54-65.

Stansell MM, Papesh MA, Srinivasan NK, Kampel SD, Belding HM, Jakien KM, Gallun FJ. (2015) The SSQ as a predictor of spatial release from masking. Paper presented at the American Auditory Society, Scottsdale, AZ.
Stebbins WC, Hawkins JE, Jr, Johnson LG, Moody DB. (1979) Hearing thresholds with outer and inner hair cell loss. Am J Otolaryngol 1(1):15-27.

Studebaker GA. (1985) A "rationalized" arcsine transform. J Speech Hear Res 28(3):455-462.

Tillman TW, Carhart R. (1966) An Expanded Test for Speech Discrimination Utilizing CNC Monosyllabic Words: Northwestern University Auditory Test No. 6. Brooks Air Force Base, TX: USAF School of Aerospace Medicine.

Turner CW, Souza PE, Forget LN. (1995) Use of temporal envelope cues in speech recognition by normal and hearing-impaired listeners. J Acoust Soc Am 97(4):2568-2576.

Turton L, Smith P. (2013) Prevalence \& characteristics of severe and profound hearing loss in adults in a UK National Health Service clinic. Int $J$ Audiol 52(2):92-97.

Van Tasell DJ. (1993) Hearing loss, speech, and hearing aids. J Speech Hear Res 36(2):228-244.

Wolfe J, Duke MM, Schafer E, Jones C, Mülder HE, John A, Hudson M. (2015) Evaluation of performance with an adaptive digital remote microphone system and a digital remote microphone audio-streaming accessory system. Am $J$ Audiol 24(3):440-450.

Won J-H, Drennan WR, Rubinstein JT. (2007) Spectral-ripple resolution correlates with speech reception in noise in cochlear implant users. J Assoc Res Otolaryngol 8(3):384-392.

Won JH, Jones GL, Moon IJ, Rubinstein JT. (2015) Spectral and temporal analysis of simulated dead regions in cochlear implants. $J$ Assoc Res Otolaryngol 16(2):285-307.

Zeng FG, Grant G, Niparko J, Galvin J, Shannon R, Opie J, Segel P. (2002) Speech dynamic range and its effect on cochlear implant performance. J Acoust Soc Am 111(1 Pt 1):377-386. 